\title{
Corrigendum
}

\section{A new tabu search heuristic for the open vehicle routing problem}

\author{
$\mathrm{Z} \mathrm{Fu}{ }^{1}, \mathrm{R}$ Eglese ${ }^{2}$ and LYO $\mathrm{Li}^{3}$ \\ ${ }^{I}$ Central South University, Changsha, PR China; ${ }^{2}$ Lancaster University, Lancaster, UK; and ${ }^{3}$ The Hong Kong \\ Polytechnic University, Hong Kong, PR China
}

Journal of the Operational Research Society (2006) 57, 1018. doi:10.1057/palgrave.jors.2602181

Correction to: Journal of the Operational Research Society (2005) 56, 267-274. doi:10.1057/palgrave.jors.2601817

Further research on the Open Vehicle Routing Problem brought to light some errors in the results reported in this paper, for which we apologize.

The computer program for the algorithm described, which sometimes produced incorrect results, has been debugged and run again on the set of test problems. In some cases, the best results previously reported have not been achieved and in other cases the new runs have produced improved results. The solutions for the best results found have been rechecked and details are available from the authors.

The runs have been carried out on a faster computer so the new run times reported here are quicker than those in the original paper.

Although the detailed results have changed, the general conclusions are not substantially altered.

A corrected version of Table 1 is given below. Only the entries in the last four columns have changed.

Table 1 Comparison of algorithms on literature problems

\begin{tabular}{|c|c|c|c|c|c|c|c|c|c|c|c|c|c|}
\hline \multirow[b]{2}{*}{ Problem } & \multirow[b]{2}{*}{$n$} & \multirow[b]{2}{*}{$L$} & \multirow[b]{2}{*}{$K_{\min }$} & \multicolumn{2}{|c|}{ Sariklis and Powell } & \multicolumn{2}{|c|}{ Brandão $(C)$} & \multicolumn{2}{|c|}{ Brandão (D) } & \multicolumn{2}{|c|}{$F u$, Eglese and $L i(R)$} & \multicolumn{2}{|c|}{ Fu, Eglese and $\mathrm{Li}(\mathrm{F})$} \\
\hline & & & & $(K) / C o s t$ & $C P U^{*}$ & $(K) / \operatorname{Cos} t$ & $C P U^{\dagger}$ & $(K) / C o s t$ & $C P U^{\dagger}$ & $(K) / \operatorname{Cos} t$ & $C P U^{\ddagger}$ & $(K) / \operatorname{Cos} t$ & $C P U^{\ddagger}$ \\
\hline $\mathrm{C} 1$ & 50 & & 5 & 488.2 & 0.22 & 438.2 & 1.7 & 416.1 & 88.8 & $416.1^{\S}$ & 0.8 & $416.1^{\S}$ & 4.1 \\
\hline $\mathrm{C} 2$ & 75 & & 10 & 795.3 & 0.16 & 584.7 & 4.9 & 574.5 & 167.5 & $567.1^{\bullet}$ & 7.8 & $569.8^{\pi}$ & 8.2 \\
\hline C3 & 100 & & 8 & 815.0 & 0.94 & 643.4 & 12.3 & 641.6 & 325.3 & 643.1 & 3.0 & 641.9 & 23.2 \\
\hline $\mathrm{C} 4$ & 150 & & 12 & 1034.1 & 0.88 & 767.4 & 33.2 & 740.8 & 870.2 & $738.9^{\bullet}$ & 6.8 & 742.4 & 47.3 \\
\hline $\mathrm{C} 5$ & 199 & & 16 & 1349.7 & 2.20 & 1010.9 & 116.9 & 953.4 & 1415.0 & $(17) / 879.0$ & 61.9 & $(17) / 879.9$ & 78.0 \\
\hline C6 & 50 & 180 & 5 & & & $(6) / 416.0$ & 1.4 & (6) $/ 413.0$ & 55.8 & $(6) / 413.0^{\S}$ & 0.6 & $(6) / 413.0^{\S}$ & 5.9 \\
\hline $\mathrm{C} 7$ & 75 & 144 & 10 & & & $(11) / 581.0$ & 3.4 & 634.5 & 123.7 & $(11) / 568.5$ & 6.3 & $(11) / 568.5$ & 6.0 \\
\hline $\mathrm{C} 8$ & 100 & 207 & 8 & & & $(9) / 652.1$ & 10.4 & (9)/644.6 & 351.7 & (9) $/ 647.3$ & 10.7 & (9)/648.0 & 36.3 \\
\hline C9 & 150 & 180 & 12 & & & (14)/827.6 & 25.2 & (13)/785.2 & 622.2 & (14)/761.3 & 46.6 & $(14) / 767.1$ & 79.3 \\
\hline $\mathrm{C} 10$ & 199 & 180 & 16 & & & (17)/946.8 & 100.1 & (17)/884.6 & 2060.3 & (17)903.1 & 51.9 & (17)/904.1 & 133.6 \\
\hline C11 & 120 & & 7 & 828.3 & 1.54 & 713.3 & 15.7 & 683.4 & 696.0 & 724.5 & 27.5 & 717.2 & 23.1 \\
\hline $\mathrm{C} 12$ & 100 & & 10 & 882.3 & 0.76 & 543.2 & 7.8 & 535.1 & 233.6 & $534.7^{\bullet}$ & 4.2 & 537.8 & 10.9 \\
\hline $\mathrm{C} 13$ & 120 & 648 & 7 & & & (11)/994.3 & 25.8 & (11)/943.7 & 401.9 & (12)/922.3 & 9.6 & (12)/917.9 & 82.1 \\
\hline $\mathrm{C} 14$ & 100 & 936 & 10 & & & (12)/651.9 & 8.1 & (11)/597.3 & 419.8 & (11)/600.7 & 2.5 & $(11) / 660.2$ & 13.0 \\
\hline F11 & 71 & & 4 & & & 179.5 & 5.7 & 177.4 & 398.1 & 177.0 & 0.4 & 178.2 & 6.3 \\
\hline F12 & 134 & & 7 & & & 825.9 & 32.7 & 781.2 & 1000.2 & $778.6^{\bullet}$ & 2.8 & $777.1^{\top}$ & 28.4 \\
\hline
\end{tabular}

*Seconds on a Pentium at $133 \mathrm{MHz}$ with $16 \mathrm{MB}$ RAM.

${ }^{\dagger}$ Seconds on a Pentium III HP Vectra VEi8 at $500 \mathrm{MHz}$.

${ }^{\ddagger}$ Seconds on a Pentium IV at $3.00 \mathrm{GHz}$ with $248 \mathrm{MB}$ RAM.

${ }^{\S}$ Means a tie with the best known solution produced by Brandão.

'Indicates that our algorithm has improved the best known solution produced by Brandão. 